
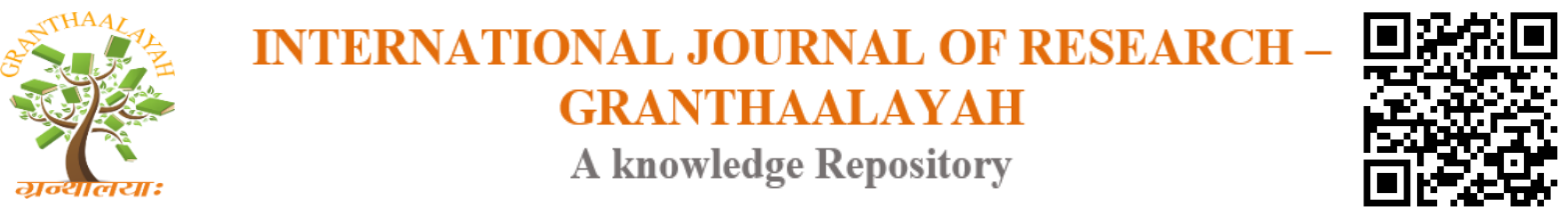

Science

\title{
APPLICABILITY OF METHODS AND TECHNOLOGIES DRIP IRRIGATION IN THE AZERBAIJAN
}

\author{
Dr. Prof. Z.H. Aliev *1 \\ ${ }^{* 1}$ Institute of Erosion and Irrigation of Azerbaijan National Academy of Sciences, Algeria
}

\begin{abstract}
With the proper conduct of the process low-intensity irrigation can not only dramatically reduce the consumption of water for irrigation agricultural crops, but also to provide the necessary climate for the plants and the supply of water and fertilizer in the required amount directly into korneobitaniya zone, which contributes to an earlier entry of plants in fruiting and increase productivity while reducing water per unit of yield and reduce production costs.

Keywords: Low-Intensity; Automation; Irrigation Technology; Regulatory; Background; Bank Data; The Controller; Connection; Object; Sensors; Etc.

Cite This Article: Dr. Prof. Z.H. Aliev. (2019). "APPLİCABİLITY OF METHODS AND TECHNOLOGIES DRIP IRRIGATION IN THE AZERBAIJAN." International Journal of Research - Granthaalayah, 7(5), 230-237. 10.29121/granthaalayah.v7.i5.2019.842.
\end{abstract}

\section{Introduction}

At the present stage of development of irrigated agriculture in our country highly efficient use of irrigated land is only possible in the implementation of modern progressive water-saving irrigation equipment and technology.

In addressing this important issue occupies a special place water management, heat, food, saline soil determined in order to maximize the cost per unit of output.

In recent years, all over the world are researching for new ways of water allocation to more fully satisfy the watering of plants.

\section{Material and Methods}

Regulation of water and associated air, heat, food, and saline soil conditions causes the development of physico-chemical and the processes occurring in the soil and determine its area. With irrigation stressful influences can lead to the destruction of the structure and permeability of 
soil aggregates that reduces soil fertility. It should be noted that certain types of irrigation have an impact not only on the ground but also on the ground layer of air, as well as directly to the plant, that is, regulate its water regime and photosynthetic processes, including by foliar feeding with water the aerial parts of the plant. It should also be noted that the introduced equipment and technology should be environmentally friendly.

Environmental safety of irrigation on the environment should be based primarily on water-saving technologies for the fullest possible use of natural precipitation, optimization and standardization of water supply, elimination of water losses in the discharge of the surface and depth filtration by creating conditions.

Purpose to rendering environmental safety of our irrigation is considered perfect new techniques and technologies of drip irrigation.

Drip irrigation technology includes water supply mode according to water consumption culture. Water supply mode depends on the norms, terms and duration of irrigation for irrigation period, humidification zone, flow, number of drippers, their locations and water-physical properties of soils.

It is known that for drip irrigation water is fed in the form of individual droplets of 1-2 $\mathrm{mm}$ in diameter or the jet directly to the local field on the surface and redistributed on it moistens the soil, mainly under the influence of capillary forces.

Irrigation technology is seen on its own and as part of the cultivation technology / crops.

The degree of perfection of technical irrigation means assessed when considering them as part of the entire irrigation industry.

Increasing the flow of irrigation equipment from the hive increasing productivity leads to an undesirable increase in the intensity of the rain, and ultimately, not to environmental acceptability, as well as from an increase in the capacity of water-supply network, increase its material and capital. The main purpose of creation and introduction of drip irrigation technology is the optimum dispersion and uniform distribution of water flow in the process of transfer to the state of the soil and air moisture. As noted above, if the question of the uniformity of distribution of conflicting opinions there is no water (the higher the coefficient of irrigation efficiency, the higher the yield), then the question of the rational degree of dispersal current water until recently there was no such unanimity. Indeed, if we compare the intensity of the water supply and the intensity of evapotranspiration, their attitude in various technologies and means of irrigation varies from 1 to 1000, with lower values correspond to low-intensity irrigation techniques.

Efficiency of irrigation technology is determined by climate, hydrological agronomic factors insufficient consideration of these factors can lead to undesirable consequences, such as runoff and depth filtration. In order to make the irrigation process more environmentally perfect, you need to choose the optimal irrigation technology that would provide the best combination of artificial and natural precipitation, and maximum use of the latter. A large number of options 
agrohydrological, climatic conditions, the characteristics of possible rainfall makes irrigation technology assessment very difficult task.

The output parameters for the calculation of the intensity of the water supply is, the duration of precipitation, evapotranspiration and intensity.

The daily water supply is determined according to the amount of evaporation from the water surface, and is calculated by the following formula:

$$
\mathrm{M}_{\text {day }}=10\left(\mathrm{E}_{\mathrm{tpd}}-\mathrm{KP}\right)=\mathrm{K}_{\mathrm{fa}}, \mathrm{m} 3 / \mathrm{ha}
$$

Where $\mathrm{E}_{\mathrm{tpd}} \mathrm{d}^{-}$evaporation of the previous day, $\mathrm{mm}$;

$\mathrm{P}$-precipitation $\mathrm{mm}$;

$\mathrm{K}$ - utilization of precipitation;

$\mathrm{K}_{\mathrm{fa}}$ - dampening factor area.

moisturizing factor of the square is defined by expression

$$
\mathrm{K}_{\mathrm{fa}}=\frac{S \cdot n}{10000}
$$

Where S - Humidification of area, $\mathrm{n}$ - Amount of drip per $\mathrm{m} 2$ of 1 ha;

The duration of the water supply is determined by the formula:

$$
\begin{gathered}
\mathrm{T}_{\text {day }}=\mathrm{M}_{\text {day }} / \mathrm{g} \cdot \mathrm{n} \\
\text { Or } \mathrm{T}_{\text {day }}=\left(\mathrm{E}_{\mathrm{tpd}}-\mathrm{K}_{\mathrm{fa}}\right) \cdot \mathrm{S} / \mathrm{g}
\end{gathered}
$$

Where $\mathrm{T}_{\text {day }}$ is the daily duration of operation of the system is quenched;

$\mathrm{M}_{\text {day }}$ - daily water supply based on the received

$\mathrm{K}_{\mathrm{fa}}-\mathrm{m} 3$ / ha

$\mathrm{g}$ - the drip rate, $1 / \mathrm{ha}$;

n quality drip per 1 ha.

In formulas 1 and 4 function $\mathrm{K}_{\mathrm{fa}}$ has significant influence to determine the technological irrigation process. The complex expresses productively used rainfall.

The utilization of precipitation (functionally depends on the intensity of the rain, the initial moisture content, soil type, slope of the terrain, the length of the rain and the surface condition of the soil.

It should be noted that if the groundwater is close to the uninhabited notice the layer of soil that the daily water supply should be determined by the following formula:

$$
\mathrm{M}_{\text {day }}-10 \mathrm{~K}_{\mathrm{fa}}\left(\mathrm{E}_{\mathrm{tpd}}(1-\mathrm{K} 2)-\mathrm{KP}\right)
$$

Where K2 utilization of groundwater 
The value of $\mathrm{K} 2$ depends on the depth observations of groundwater, water-physical properties of the soil and the depth distribution of the root system of plants. We offer two methods of the process of irrigation crops.

The first method is based on the traditional method.

According to the method in the absence of rain watersupplies replenished daily to the desired value in accordance with the diurnal day prior to evaporation. It uses evidence GTI-3000.

At the beginning of the irrigation season, and after every rain measured initial moisture content of the soil-or how accurate enough method.

The second method is also based daily replenishment of soil moisture reserves for the required value according to evaporation in the previous day in terms of isparometra GGI-3000.

Soil moisture (initial moisture content) is determined by calculation. This method is based on the theoretical and experimental curves for determining the effectiveness of fallen rain. Used for the calculation takes into account the dependence of the water and soil properties (the ability to elicit), and rain parameters (intensity, duration).

The initial parameters of the technological process of watering, characterizing soil conditions, moisture content is calculated soil. The moisture content of the soil layer is calculated, corresponding to $100 \%$ of $\mathrm{NV}$ is determined from the expression:

$$
\mathrm{P}_{\mathrm{nv}}=100 \mathrm{H} \cdot \mathrm{N} \beta_{\mathrm{nv}}
$$

Where $\mathrm{H}$ is the calculated volume weight of the soil layer, $\mathrm{t} / \mathrm{cm}^{2}$;

$\mathrm{N}$ is the depth of the active layer of soil $\mathrm{m}$;

ß- soil moisture as a percentage by weight of dry soil.

If we consider that to be determined by the following formula for the heavy loam soil moisture equal to the lower limit of $\beta_{\min }=80 \% \mathrm{NV}$, and the upper limit of $100 \% \beta_{\max } \mathrm{NV}$, the optimal conditions for these soil moisture:

$$
\beta_{\mathrm{opt}}=\underline{\beta_{\max }+\beta_{\min }}
$$

\section{2}

That is for the heavy loam soil is optimal humidity is $90 \% \mathrm{NV}$. Then the calculated moisture content of the soil layer, the corresponding optimum moisture $\beta_{\mathrm{opt}}=90 \mathrm{NV}$ gets the following form:

$$
\mathrm{P}_{\mathrm{opt}}=0.9 \mathrm{P}_{\mathrm{nv}}
$$

The same method can be used to determine the optimum moisture content of the soil layer is calculated for other soil conditions (light, medium, etc.). 
Irrigation technology under drip irrigation should be carried out as follows:

At the beginning of the growing season determines the initial moisture content and the corresponding water content $B_{o} P_{o}$ in the settlement layer of soil, and the soil moisture content is measured by one of the exact methods.

If it turns out that $\mathrm{P}_{\mathrm{o}}<\mathrm{H} 2$ schopt the beginning of the vegetation watering spend before watering irrigation norm

$$
\mathrm{m}=\mathrm{K}_{\mathrm{fa}}\left(\mathrm{P}_{\mathrm{opt}}-\mathrm{P}_{\mathrm{o}}\right)
$$

If $\mathrm{P}_{\mathrm{o}}>\mathrm{P}_{\mathrm{opt}}$ the daily irrigations carried out after all it is the soil moisture reserves $\mathrm{P}_{\mathrm{o}}$ will not fall to the desired level.

Irrigations (daily rate and duration of watering) is carried out in terms of evapotranspiration. For this purpose, the IP section GGI-3000.

If the previous day evaporated from the soil, for example, $5 \mathrm{~mm}$ of moisture, then the day in question in drip irrigation is applied to the soil 2.5 (25 $\mathrm{m} 3 / \mathrm{ha})$, and finely divided moisture and other irrigation $5 \mathrm{~mm}(50 \mathrm{~m} 2 / \mathrm{ha})$. This is because under drip irrigation is not the whole territory is occupied by crops. Only locally irrigated areas close to the plant root system. The length of the system is determined by nomogram 1 .



Figure 1: Nomogram for determining the length of the system work

During the without rainy season it is repeated every day.

In the rain, in two or three days after the rain, soil moisture is measured on the basis of what this. The difference between the $\mathrm{P}_{\mathrm{o}}$ and $\mathrm{P}_{\mathrm{opt}}$ or soil to make a one-time rate of watering.

$\mathrm{P}_{\text {opt }}-\mathrm{P}_{\mathrm{o}}\left(\mathrm{P}_{\mathrm{opt}}-\mathrm{P}_{\mathrm{o}}\right)$ or until the next growing watering should wait until such a day from the soil to evaporate the difference, that is:

$$
\mathrm{P}_{\text {opt }}-\mathrm{P}_{\mathrm{o}}\left(\mathrm{P}_{\mathrm{opt}}>\mathrm{P}_{\mathrm{o}}\right)
$$

Evapotranspiration measurements and duration of daily watering entered in a special magazine, where the parameters of technological irrigation process is determined by nomogram 1-4. 


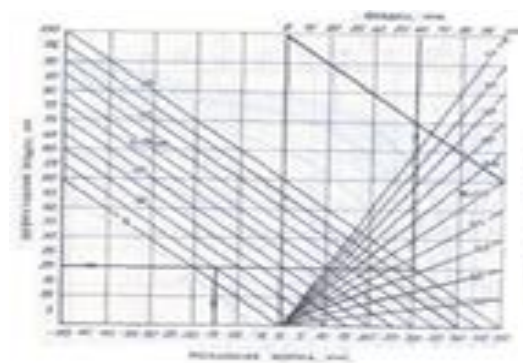

The nomogram for determining irrigation

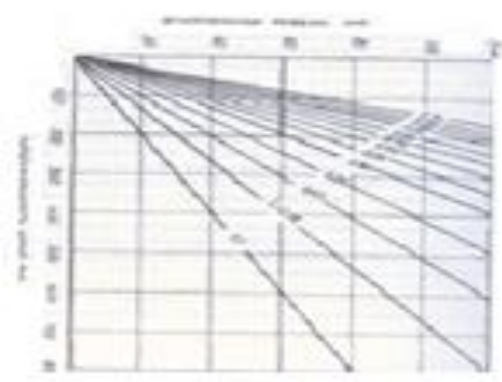

Fiq.3 The nomogram for determining the rain

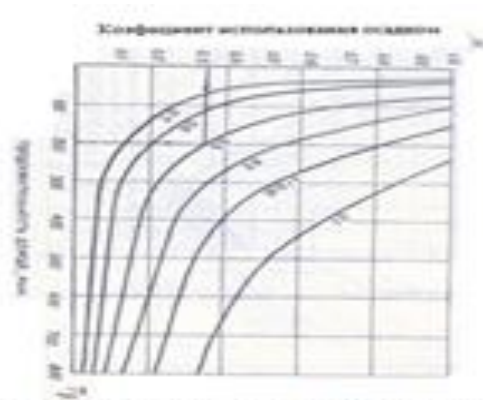

Fiq.4 The nomogram for determining the utilization rate of precipitation

These nomograms developed for heavy loam soil. The slope of the terrain is used to zero. In the construction of the nomogram for determining irrigation norms $\mathrm{m}$ soil moisture coefficient was taken equal to 1 . This expands the scope of this nomogram and other irrigation methods. With drip irrigation resulting nomogram normal watering adjust the size of the soil moisture factor, $\mathrm{m}=\mathrm{m}$. $\mathrm{K}_{\mathrm{fa}}$

Nomograms Figure 1-4 allow irrigators without making measurements of soil moisture to determine the parameters of the technological process of irrigation.

To reliably determine the process of watering should consider some of the specific case, that is not rainy and rainy periods.

Without watering the rainy period is carried out as follows.

During the vegetation irrigation, irrigation rate is equal to the total evaporation of the previous day, taking into account the $\mathrm{E}_{\mathrm{tpd}}$ ratio of soil moisture. The coefficient of soil moisture is sprinkling $\mathrm{Km}=1$, and under drip irrigation.

$\mathrm{K}_{\mathrm{fa}}=0.4$, that is, under drip irrigation is not the whole area is moistened so pridozhdevanii the daily norm $\mathrm{m}=\mathrm{E}_{\mathrm{tpd}}$ under drip irrigation at $\mathrm{K}_{\mathrm{fa}}=0.4 \mathrm{E}_{\mathrm{tpd}}$.

The rain during the payment process parameters are as follows:

With the help of GTI-3000 is determined by the amount of precipitation, evaporation of the previous day and the duration of the $\mathrm{E}_{\mathrm{tpd}} \mathrm{T}_{\mathrm{e}}$ rain recorder via humidity or temperature of the air (beginning and end of rain on lek). Knowing the amount of precipitation and the duration of $\mathrm{T}_{\mathrm{e}} \mathrm{ni}$ rain can easily determine the average intensity of the rain;

$$
\mathrm{L}_{\mathrm{m}} \text { int }=\frac{h}{t_{g}}
$$

According to well-known $\mathrm{T}_{\mathrm{e}}$ and $\mathrm{P}$ using the formula (6.10) can build a nomogram rain intensity. Figure 1 shows a nomogram for determining the intensity of the rain. According to well-known $T_{e}$ and intensity of rain Iunt nomogram Fig. 2 determine the utilization of rainfall in. Next, using the nomogram in Fig. 3 on the known values of liters, $\mathrm{E}_{\mathrm{tpd}}$ and $\mathrm{P}$ find the desired rate of watering places for soil moisture to the optimum level. 


\section{Results and Discussion}

Usually rains the current value of moisture reserves in the soil becomes more than required, since before the rain, soil moisture is maintained at the optimum level. The problem is reduced to determining the current value of soil moisture reserves after the rain. At the same time soil moisture reserves rain increased by the amount of layer effectively sunken precipitation. Regular watering the vegetation is made after evaporation from the soil effective natural precipitation. Thus, the proposed method determines the parameters of the technological process of irrigation, allow for practical application of the most productive use of precipitation. It is sufficient to identify the needs of irrigation rate and assign the timing and duration of the system.

The above recommendations for the operation of drip irrigation systems and irrigation technology can be used effectively in a variety of climatic conditions of Azerbaijan.

\section{Conclusions}

Providing substantial growth and development of crops on account of the introduction of drip irrigation systems with the use of mineral fertilizers together with the irrigation water in the favorable conditions of Azerbaijan. Adding mineral fertilizers through drip system much more efficiently than any other method. What the proposed drip irrigation systems compared to traditional dominant republic irrigation methods is very promising, thus allowing plant irrigation water continuously during their growing season, taking into account the conservation of ecological equilibrium of the environment.

\section{Findings}

Ensuring substantial growth, development and crop on account of the introduction of drip irrigation systems with the ability to use mineral fertilizers together with irrigation water conditions are favorable in Azerbaijan. Adding mineral fertilizer through drip system much more efficiently than any other method. What is proposed by drip irrigation systems as compared to the traditional dominant method of irrigation in the country is very promising, thus allowing plant irrigation water continuously during their growing season with a view to preserving the ecological balance of the environment.

\section{References}

[1] B.H Aliev, Aliev Z.H "Irrigation Engineering for farmer and farms of Azerbaijan" monograph //, Publishing house "Azerneshr" Baku, 1998.113

[2] B.H Aliev, Aliev Z.H Zoning of territory of Azerbaijan Republic on choosing advanced irrigation techniques. / Monograph, Publishing house "Ziya". Baku, 2001. 297 p.

[3] B.H Aliev, Aliev Z.H Irrigated agriculture in the mountain and foothill regions of Azerbaijan .// Monograph Publishing house "Nurlan Zia EPG Ltd", Baku, 2003. 330 p.

[4] Aliev B.H, Aliev Z.H and others Techniques and technology few intensive irrigations in condition of the mountain region Azerbaijan.// Publishers "Elm", Baku, 1999, p. 220.

[5] Aliev B.H, Aliev Z.H. The premises about the most important problem of the agriculture in provision water resource mountain and foothill regions Azerbaijan, // J. AAS, \# 1-3, Baku, 2007, p.179-182. 
[6] Aliev B.H, Aliev Z.H. The premises of the decision of the problems moisture provides agriculture cultures production in mountain and foothill region Azerbaijan.// The works SRI "Erosions and Irrigations". Baku, 1999, p.125-129.

[7] Guseynov NM Ways to improve the efficiency of use of irrigated land, improved technologies and methods for irrigation of agricultural crops in Azerbaijan. // Report on the degree of competition. C. c. D. on the basis of works, Baku, 1969 s.214-230.

[8] Mezhdunarodny Center C / X Research in the dry in the Dry Areas (ICARDA) Irrigation regime and monitoring equipment .// Edited U.Umarova and A Karimov. Taraz: IC "AQUA", 2002, 128 p.

[9] V.F.Nosenko Irrigation in the mountains .// Publishing House "Kolos" Moscow 1981,143 p.

\footnotetext{
*Corresponding author.

E-mail address: zakirakademik@ mail.ru
} 